\title{
Is AM1.5 applicable in practice? Modelling eight photovoltaic materials with respect to light intensity and two spectra
}

\author{
J. F. Randall", J. Jacot \\ Laboratoire de Production Microtechnique, \\ IPR, STI, Ecole Polytechnique Fédérale de \\ Lausanne, CH-1015 Lausanne, Switzerland
}

\begin{abstract}
Solar cell comparison is generally based on an arbitrary maximum terrestrial intensity and spectra (of 1 sun, $1000 \mathrm{~W} / \mathrm{m}^{2}$ ) at $25^{\circ} \mathrm{C}$ perpendicular to the cell plane [1] referred to by specialists as AM1.5. In practice, no solar cell experiences such conditions, yet few alternative bases for comparison exist [2]. Our interest in this paper is to explore the correct design of indoor Photovoltaic (IPV) products. Given that the indoors, when compared with the outdoors, are characterised by much lower radiant energy intensities, various spectra (including artificial light sources), complete comparison data for indoor conditions is not freely available. More general level reports have been published [3-6].

21 different solar cells representing 8 different Photovoltaic material technologies are reproducibly electrically characterised under laboratory based simulated AM1.5 (1 sun; solar spectrum) from $1000 \mathrm{~W} / \mathrm{m}^{2}$ intensity down to the $0.1-1 \mathrm{~W} / \mathrm{m}^{2}$ decade. Some were measured under an artificial light source (fluorescent tube) in the $1-10 \mathrm{~W} / \mathrm{m}^{2}$ decade.
\end{abstract}

The results are used to validate a phenomenologically based model.

Keywords: Intensity; Photovoltaic Technology; Spectra; Survey

\section{Introduction}

The inexorable growth in low power micro-electronic devices such as sensors and MEMS (Micro Electro Mechanical Systems) is an opportunity for increasing the use of PV (Photovoltaics) especially for indoor applications. Photovoltaic modules may be used to partially or completely source the energy required for the functioning of such systems. For information, indoor consumer PV represented $4 \mathrm{MW}_{\mathrm{P}}$ in 1997. It is of note that the 1 sun efficiency reference of such statistics is misleading for indoor products, as electrical efficiency is much less important indoors. This is because the end-user decision to purchase an indoor PV (IPV) product is not related to the solar cell electrical efficiency but

\footnotetext{
* Corresponding author: Telephone: ++ 4121693 5945, Fax: ++ 4121693 3891, E-mail address: Julian.Randall@epfl.ch (J.F. Randall)
} 


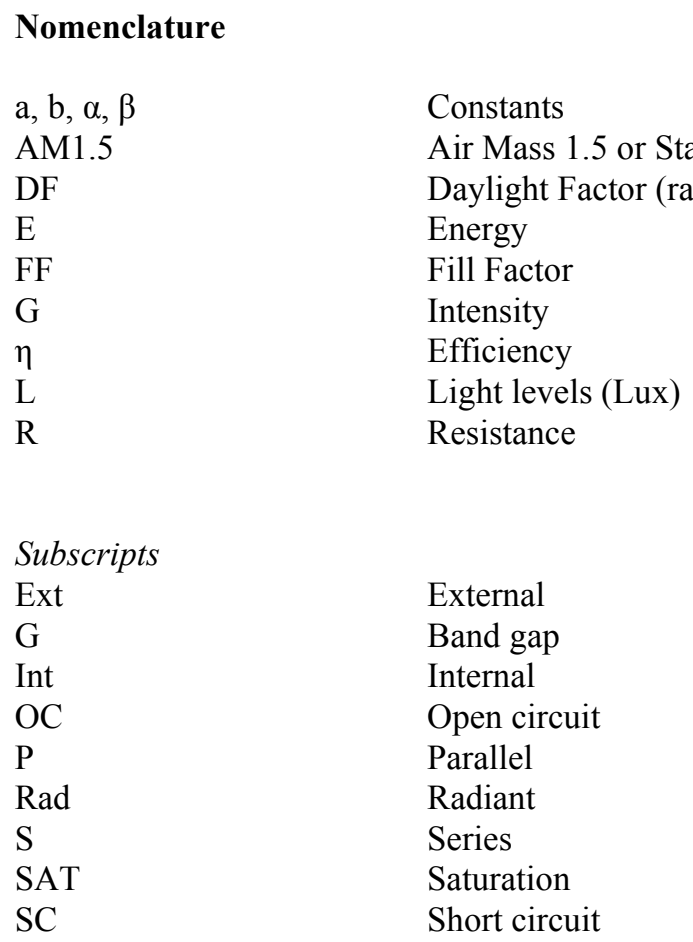

rather to such benefits as reduced reliance on batteries ("plug and forget") and increased reliability i.e. a correctly designed and used IPV system can run longer without user intervention than when powered by batteries alone. From an environmental responsibility perspective, reducing battery waste is also laudable.

This paper forms part of a wider project to examine how to extend IPV use beyond the solar calculators and watches to which we are already accustomed. Whilst data is available to the IPV designer, such as PV materials available, cost, colour, surface areas, 1 sun voltage/current and so on, there remain areas of missing and yet salient information. One of these areas is comparable electrical performance at the light intensities and spectra typical of the indoor environment $\left(<10 \mathrm{~W} / \mathrm{m}^{2}\right.$ at $>1 \mathrm{~m}$ from artificial light source or $<100 \mathrm{~W} / \mathrm{m}^{2}$ at $>1 \mathrm{~m}$ from window). Another is a suitable model to predict performance under these conditions. Some comparisons exist for indoor [3-6] and outdoor conditions [7-10]. However, none cover the range of PV technologies, intensities and spectra of this paper.

Using solar cells indoors is both different from outdoors (less variation of temperature, much less intensity $\left\{1-10 \mathrm{~W} / \mathrm{m}^{2}\right.$ range rather than $100-1000 \mathrm{~W} / \mathrm{m}^{2}$ range outside $\}$, further spectra, variation of performance with intensity) and similar (importance of cell orientation with respect radiant energy source/s, impact of obstacles). These issues are more easily understood when one considers those who influence the built environment namely the creators (architects and lighting engineers in the case of IPV) and end-users. Architects when designing in practice try to achieve a balance between a number of factors including safety, cost and comfort (light, temperature, aesthetics etc.). Chief among these for IPV 
needs is the light levels which they attempt to maintain as uniform as possible, although this is rarely possible by daylight alone. The reason for this can be seen in Figure 1, showing typical values of Daylight Factor (DF), which are quite low (a few percent). DF is calculated by the ratio of $\mathrm{L}_{\text {int }} / \mathrm{L}_{\text {ext }}$, where $\mathrm{L}_{\mathrm{int}}$ and $\mathrm{L}_{\text {ext }}$ are the light levels in Lux indoors and outdoors respectively. Note the rapid decrease of DF between window and opposite wall to which the human eye adapts imperceptibly.

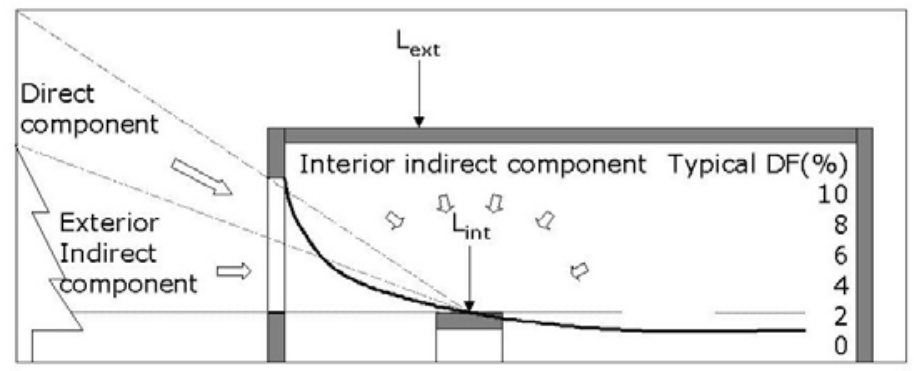

Figure 1: Daylight factor components and typical values

Lighting engineers aim to fulfil a specification from an architect that complements the daylight component, with the aim of providing whatever artificial lighting will be required. Users are a stochastic element in any system that can be modelled. They can have a significant impact on the available light by the fittings and furniture they require as well as their use-pattern, e.g. how they use lighting and blinds.

These 3 groups of actors seek a perceived (or photometric) result, whilst PV collects radiant energy. For this paper, the word "light" is used to describe photometric radiation whilst "radiant energy" $\left(\mathrm{E}_{\mathrm{rad}}\right)$ is used for the wider bandwidth radiation to which solar cells are sensitive. Our experiments measuring $\mathrm{E}_{\mathrm{rad}}$ indoors have confirmed similar trends to those found for light (or DF) shown in Figure 1.

In order to increase the range of experiments, simulation can be performed with less resources (time, number of sensors, varying less parameters at a time i.e. daylight variation during measurement). The choice of software should be made by prioritising physical accuracy and as such "Radiance" [11] is ideal. The solar cell data presented in this paper when combined with predicted $\mathrm{E}_{\mathrm{rad}}$ data allow the cumulative charge $(\mathrm{Ah})$ available to be calculated.

Following this section the method used for testing the cells is described (Experimental Procedure) as well as the related findings (Results). In the Model Presentation section, a model is explained and compared with the results. Other issues of interest are reviewed in the Discussion.

\section{Experimental Procedure}

The solar cells were characterised under indoor conditions which departed as little as possible from Standard Test Conditions (STC) [1]. The current/voltage (I/V) characteristics were taken in the standard way for the 21 samples in Table 1 using a Wacom solar simulator as previously described [6]; the $\mathrm{E}_{\mathrm{rad}}$ intensity was controlled with one or more wire mesh filters between the $\mathrm{E}_{\mathrm{rad}}$ source and the sample which at maximum filtration 
reduced the $1000 \mathrm{~W} / \mathrm{m}^{2}$ to approximately $0.8 \mathrm{~W} / \mathrm{m}^{2}$. At each level of intensity, an I/V curve was recorded, as well as the resistances at short circuit, $\mathrm{R}_{\mathrm{SC}}$ and open circuit, $\mathrm{R}_{\mathrm{OC}}$.

Some samples were tested in the same way other than a different light source was used (fluorescent Philips Ecotone PL-L, 830/4P HF, 40W) to be representative of artificial light sources. The intensity from this source on the solar cell was varied over the $1-5 \mathrm{~W} / \mathrm{m}^{2}$ by controlling the distance ( $>1 \mathrm{~m}$ range) between the source and the solar cell under test.

The indoor environment typically has a smaller temperature range than outdoors, so all experiments were performed at a fixed temperature $\left(22^{\circ} \mathrm{C}+/-3\right)$. As solar cell efficiency is affected by temperature, this contributed to reducing the uncertainty related to varying more than one variable at a time, often found in outdoor comparative testing [7-10].

Table 1: Technologies and sources of the 21 cells tested showing whether the manufacturer was a laboratory or industry, the active area and number of cells in the module of each sample tested

\begin{tabular}{|l|l|l|c|c|c|}
\hline \multicolumn{1}{|c|}{ Technological Classification } & \multicolumn{1}{|c|}{ Supplier or Laboratory name } & \multicolumn{1}{c|}{ Cell Code } & $\begin{array}{c}\text { Indu. }=\text { I } \\
\text { Labo }=\text { L }\end{array}$ & $\begin{array}{c}\text { Active } \\
\text { Area }\left(\mathbf{c m}^{2} \text { ) }\right.\end{array}$ & $\begin{array}{c}\text { No. of cells } \\
\text { in module }\end{array}$ \\
\hline Silicon (crystalline) & BP Solar (via IWS) & XSi-BP & I & 9.36 & 1 \\
\hline Silicon (crystalline LGBC) & BP Solar, UK & XSi-LGBC & I & 0.90 & 1 \\
\hline Silicon (crystalline) & Spacecells, Edmund Scientifc, US & xSi-EdSi & I & 0.38 & 1 \\
\hline Silicon (crystalline) & Unknown (via Distributor) & xSi-Dist & I & 10.95 & 1 \\
\hline Silicon (polycrystalline) & MAIN, TESSAG, D & pSi-MAIN & I & 12.47 & 1 \\
\hline Silicon (polycrystalline) & EFG, TESSAG, D & pSi-EFG & I & 10.25 & 1 \\
\hline Silicon (polycrystalline) & Unknown (via Distributor) & pSi-Dist & I & 2.88 & 1 \\
\hline III-V cells (GaAs) & NREL, Golden, CO, US & 3-5-NREL & L & 0.25 & 1 \\
\hline Polycrystalline thin film (CdTe) & CdTe-Mats & I & 5.80 & 5 \\
\hline Polycrystalline thin film (CdTe) & Matsushita / Panasonic, J & CdTe-Parm & L & 0.79 & 1 \\
\hline Polycrystalline thin film (CIGS) & Parma University, I & CIGS-ZSW & L & 0.46 & 1 \\
\hline Other (GalnP) & ZSW, Stuttgart University, D & GIP-NREL & L & 0.25 & 1 \\
\hline Amorphous Silicon & NREL, Golden, CO, US & aSi-Tess & I & 4.95 & 5 \\
\hline Amorphous Silicon & TESSAG, Putzbrunn, D & aSi-Sany & I & 3.71 & 4 \\
\hline Amorphous Silicon & Sanyo Electric, Hyogo, J & aSi-Sole & I & 1.76 & 3 \\
\hline Amorphous Silicon & Solems, Paris, F & aSi-VHF & L & 3.36 & 4 \\
\hline Amorphous Silicon & VHF Technologies, Le Locle, CH & aSi-Sino & I & 1.26 & 4 \\
\hline Amorphous Silicon & Sinonar Corporation, Taipei, TW & aSi-BP & I & 0.20 & 1 \\
\hline Photochemical (Nanocrystalline dye) & Millenium, BP Solar & PC-GCSA & L & 1.00 & 1 \\
\hline Photochemical (Nanocrystalline dye) & Greatcell SA, Yverdon, CH & PC-ICP2 & L & 0.90 & 1 \\
\hline Multijunction cell (GaAs-GalnP tandem) & EPFL ICP2, Lausanne, CH & MJ-NREL & L & 0.25 & 1 \\
\hline
\end{tabular}

\section{Results}

Samples were accepted from across the quality range from cheap commercial cells up to high performance prototypes. No distinction was made with regard whether they were designed for indoor or outdoor use. The chief acceptance criteria was that they have an active area suitable for IPV which was taken in this case as being less than $5 \mathrm{~cm} \times 5 \mathrm{~cm}$ $\left(25 \mathrm{~cm}^{2}\right)$.

Figure 2 (left graphs) shows that solar cell efficiency in the highest intensity decade, 100$1000 \mathrm{~W} / \mathrm{m}^{2}$, varies less than in the lower decades. The ranking by 1 sun efficiency is almost completely maintained down to $200 \mathrm{~W} / \mathrm{m}^{2}$. For intensities below $100 \mathrm{~W} / \mathrm{m}^{2}$ (see Figure 2 right graphs), which are typical of indoor conditions, a much more marked change is found and the ranking by technology is altered when one reaches the lowest intensities so that some of the highest performing cells at 1 sun were the weakest at $1 \mathrm{~W} / \mathrm{m}^{2}$. 

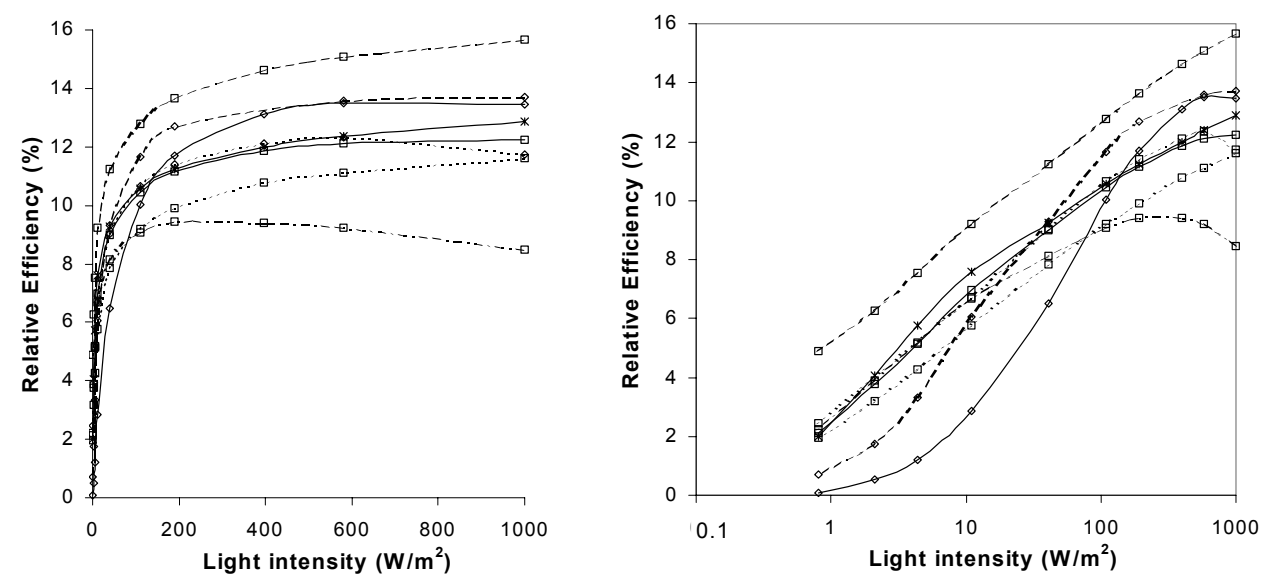

\begin{tabular}{|c|c|c|c|}
\hline$\because \mathrm{xSi-BP}$ & $-\square-\mathbf{X S i}-$ LGBC & $\cdots \boxminus \cdots \mathbf{x S i}-\mathbf{E d S i}$ & $-\cdot \theta \cdot-\mathbf{X S i} \mathbf{D}-\mathbf{D}$ ist \\
\hline$\rightarrow$ pSi-MAIN & $-\infty-$ pSi-EFG & $\cdots \otimes$ pSi-Dist & * CIGS-ZSW \\
\hline
\end{tabular}
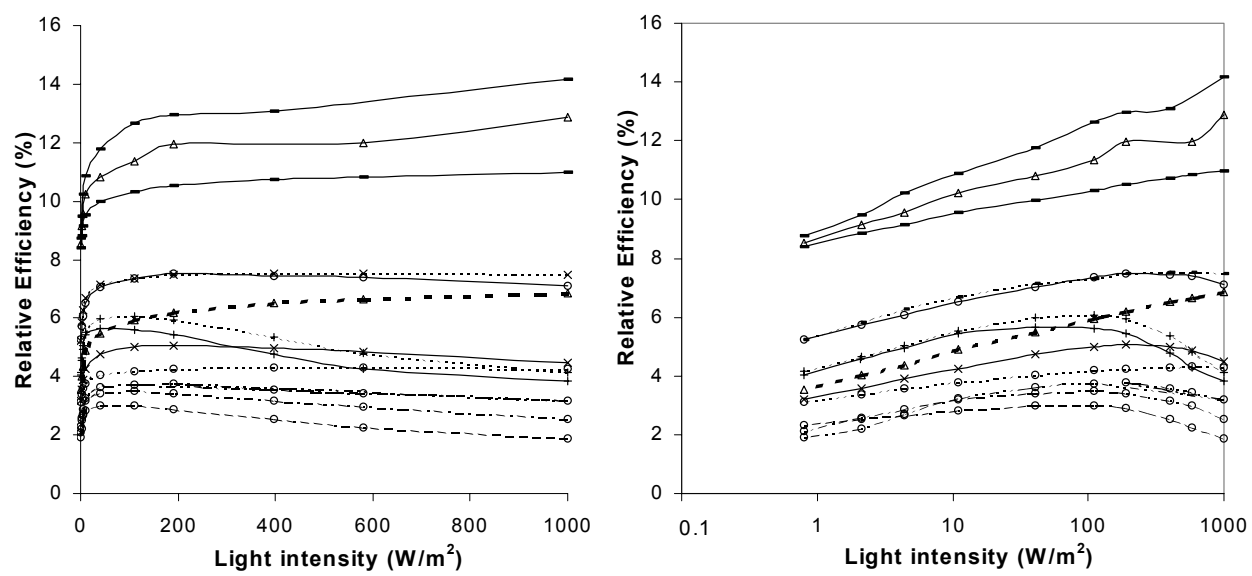

\begin{tabular}{|c|c|c|c|c|}
\hline - MJ-NREL & $\triangle 3-5-N R E L$ & - GIP-NREL & $\star$ CdTe-Mats & $x$ CdTe-Parm \\
\hline $\begin{array}{l}\multimap \text { aSi-Tess } \\
-\ldots-a S i-B P\end{array}$ & $\begin{array}{l}-\ldots-\text { aSi-Sany } \\
\ldots+\ldots \text { PC-GCSA }\end{array}$ & $\begin{array}{r}\ldots \text { a } \\
\ldots \text { aSi-Sole } \\
\text {, PC-ICP2 }\end{array}$ & $-\cdot \theta--\mathbf{a S i} \mathbf{V H H F}$ & -a-aSi-Sino \\
\hline
\end{tabular}

Figure 2: Efficiency of all samples under wire-mesh filtered AM1.5 $\left(1000 \mathrm{~W} / \mathrm{m}^{2}\right)$ showing same results vs. intensity on the base 10 scale (left) and natural logarithm (right). The log scale slope has been used to sort the results between the top and bottom graphs (see phenomenological model). 

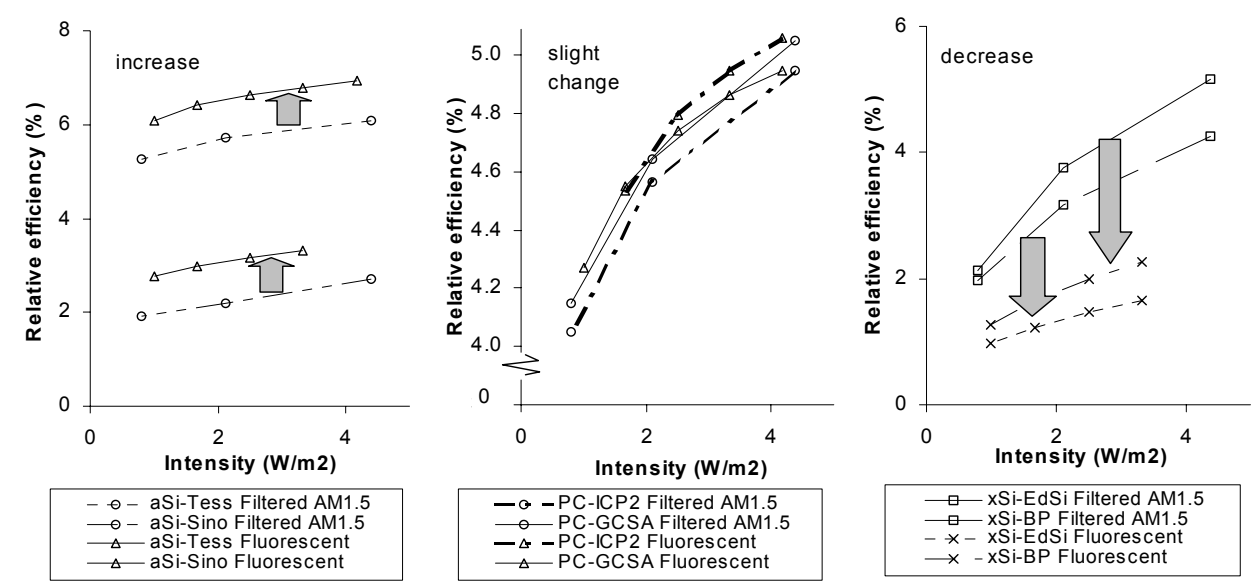

Figure 3: Efficiency difference going from filtered AM1.5 to the fluorescent spectrum for 2 samples of 3 PV technologies: amorphous Silicon single cells (left), dye cell (middle) and crystalline Silicon (right)

The graphs in Figure 3 compare the efficiencies under filtered AM1.5 with those found under the fluorescent source for selected samples representing 3 technologies. The fluorescent intensity was measured using a Lux meter and then converted to $\mathrm{W} / \mathrm{m}^{2}$ using the (simplified) relationship:

$$
\frac{E_{\text {rad }}(\text { Lux })}{120,000}=\frac{E_{r a d}\left(\mathrm{~W} / \mathrm{m}^{2}\right)}{1,000}
$$

or:

$$
E_{r a d}\left(W / m^{2}\right)=\frac{E_{r a d}(\operatorname{Lux})}{120}
$$

\section{Model Presentation}

Efficiency $(\eta)$ is calculated as follows:

$\eta=\frac{F F \times I_{S C} \times V_{O C}}{G}$

where $I_{S C}$ is short circuit current, $V_{\mathrm{OC}}$ is open circuit voltage, $\mathrm{G}$ is intensity (equal to $\mathrm{E}_{\mathrm{rad}}$ ) and FF (Fill Factor) is the ratio of the maximum power output of the cell to the product of $\mathrm{I}_{\mathrm{SC}}$ and $\mathrm{V}_{\mathrm{OC}}$. For the samples in Figure 4 it can be seen that FF is approximately constant in the range $1-100 \mathrm{~W} / \mathrm{m}^{2}$. This was found to be relatively valid for all samples, for example the FF maximum - FF minimum for the range $8-100 \mathrm{~W} / \mathrm{m}^{2}$ was $2 \%$ for $\mathrm{CdTe}, 5 \%$ for a-Si and dye cells, $22 \%$ for poly-crystalline Silicon and $23 \%$ for mono-crystalline Silicon. Moreover, it also well known that $\mathrm{I}_{\mathrm{SC}}$ is directly proportional to $\mathrm{G}$; in this case $\alpha \mathrm{G}$ is used instead of $\mathrm{I}_{\mathrm{SC}}$ where $\alpha$ is a constant:

$$
I_{s c}=\alpha G
$$




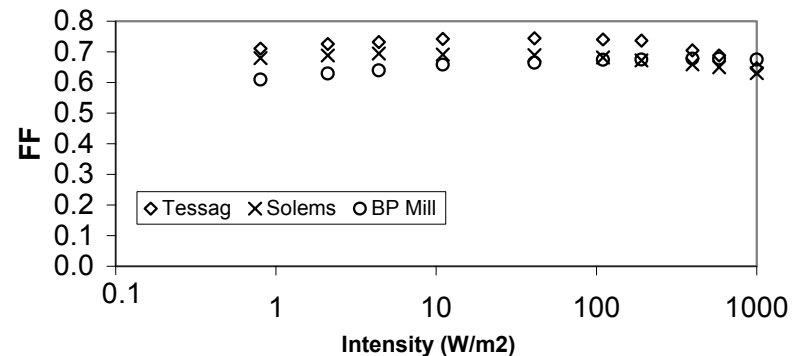

Figure 4: FF vs. Intensity on base 10 log. scale for selected amorphous Silicon samples Given that $\mathrm{V}_{\mathrm{OC}}$ has the following relationship with $\mathrm{I}_{\mathrm{SC}}$ :

$$
V_{O C} \cong \frac{k T}{q} \ln \left(\frac{I_{S C}}{I_{S A T}}\right)
$$

where $\mathrm{I}_{\mathrm{SAT}}$ is the saturation current and $\mathrm{kT} / \mathrm{q}$ is the thermal voltage, substituting (4) and (5) into equation (3), it can be found that:

$$
\eta \cong F F \cdot \alpha \cdot \frac{k T}{q}\left(\ln G+\ln \alpha-\ln I_{s}\right)
$$

$\mathrm{I}_{\mathrm{SAT}}$ can be found [12] \& [13] using the approximate formula:

$$
I_{S A T} \cong \beta \exp \left(-\frac{E_{g}}{k T}\right)
$$

where $E_{g}$ is the band gap, $\beta$ is relatively constant and temperature, $T(K)$ is held constant for the experiments, equation (6) becomes:

$$
\eta \cong F F \cdot \alpha \cdot \frac{k T}{q}\left(\ln G+\ln \alpha-\ln \beta+\frac{E_{g}}{k T}\right)
$$

which in the form:

$$
\eta=a \ln G+b
$$

has:

$a=F F \cdot \alpha \cdot \frac{k T}{q}$

and:

$$
b=F F \cdot \alpha \cdot \frac{k T}{q}\left(\ln \alpha-\ln \beta+\frac{E_{g}}{k T}\right)
$$

From the right hand graphs of Figure 2, it can be seen that the overall trend is a straight line on a logarithmic scale. This is particularly the case in the range $1-100 \mathrm{~W} / \mathrm{m}^{2}$ and for the lower right hand graph. In order to show this more clearly, Figure 5 provides a 
magnification of the experimental data (points) and their fit with equation 9 (lines) over the range $1-100 \mathrm{~W} / \mathrm{m}^{2}$. This latter relationship was applied to the data for all samples in the range $0.8-100 \mathrm{~W} / \mathrm{m}^{2}$ and the results shown in Table 2 suggest a satisfactory fit (average linear correlation coefficient $\mathrm{R}^{2}$ of 0.98 ).

An ideal cell for IPV use therefore has as low a value for $a$ and as high a value for $b$ as possible, as displayed by those samples that perform best in our experiments under indoor light conditions (e.g. Ga compounds). These two effects can be summarised by altering equation 9 as follows:

$$
\eta=b\left(\frac{a}{b} \ln G+1\right)
$$

which gives:

$$
\frac{a}{b}=\frac{1}{\ln \alpha-\ln \beta+\frac{E_{g}}{k T}}
$$

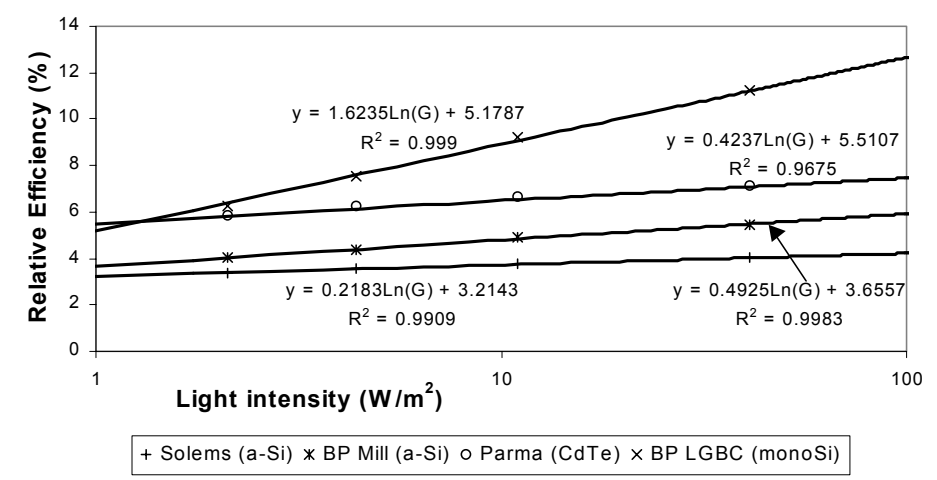

Figure 5: A magnification of Fig. 2 (r.h.s.) for selected samples compared with the fit of equation 9 
Table 2: Phenomenological model (equation 9) parameters over in $1-100 \mathrm{~W} / \mathrm{m}^{2}$ range

\begin{tabular}{|c|c|c|c|c|c|c|c|c|c|c|c|}
\hline Cell code & $a_{A M}$ & $\boldsymbol{b}_{\mathrm{AM}}$ & $\mathrm{R}^{2}$ & $a_{A M} / b_{A M}$ & $\sim \mathrm{Eg}$ & Cell code & $a_{\mathrm{AM}}$ & $b_{\mathrm{AM}}$ & $\mathrm{R}^{2}$ & $a_{A M} / b_{A M}$ & $\sim \mathrm{Eg}$ \\
\hline pSi-EFG & 2.33 & 0.48 & 0.99 & 4.87 & 1.10 & aSi-Sino & 0.40 & 2.06 & 0.95 & 0.19 & 1.70 \\
\hline pSi-MAIN & 2.05 & $(-) 0.84$ & 0.92 & 2.43 & 1.10 & aSi-VHF & 0.33 & 2.30 & 0.96 & 0.14 & 1.70 \\
\hline xSi-EdSi & 1.50 & 2.16 & 1.00 & 0.69 & 1.10 & aSi-BP & 0.49 & 3.66 & 1.00 & 0.13 & 1.70 \\
\hline xSi-BP & 1.71 & 2.58 & 1.00 & 0.66 & 1.10 & CdTe-Mats & 0.38 & 3.30 & 1.00 & 0.11 & 1.40 \\
\hline CIGS-ZSW & 1.73 & 2.83 & 0.98 & 0.61 & 0.90 & PC-IPC2 & 0.42 & 4.25 & 0.95 & 0.10 & - \\
\hline pSi-Dist & 1.67 & 2.80 & 1.00 & 0.60 & 1.10 & PC-GCSA & 0.40 & 4.37 & 0.96 & 0.09 & - \\
\hline xSi-Dist & 1.40 & 2.88 & 0.99 & 0.48 & 1.10 & MJ-NREL & 0.74 & 9.02 & 0.99 & 0.08 & - \\
\hline \multirow[t]{2}{*}{ xSi-LGBC } & 1.62 & 5.18 & 1.00 & 0.31 & 1.10 & aSi-Tess & 0.43 & 5.41 & 0.99 & 0.08 & 1.70 \\
\hline & & & & & & CdTe-Parm & 0.42 & 5.51 & 0.97 & 0.08 & 1.40 \\
\hline \multicolumn{6}{|l|}{$\mathrm{Nb}:$} & aSi-Sany & 0.17 & 2.39 & 0.98 & 0.07 & 1.40 \\
\hline \multicolumn{6}{|c|}{$a_{\mathrm{AM}} \& b_{\mathrm{AM}}$ are values of $a \& b$ under AM1.5 source } & aSi-Sole & 0.22 & 3.21 & 0.99 & 0.07 & 1.40 \\
\hline \multicolumn{6}{|c|}{ Cell codes are defined in Table 1} & 3-5-NREL & 0.57 & 8.72 & 0.98 & 0.07 & 1.50 \\
\hline \multicolumn{6}{|c|}{ Average $R^{2}$ for all 21 samples 0.98} & GIP-NREL & 0.36 & 8.59 & 1.00 & 0.04 & 1.40 \\
\hline
\end{tabular}

Equation 13 indicates that with $\alpha, \beta$ and T constant, $a / b$ is inversely related to band gap. In Table 2 , the values of $a / b$ are shown for all samples tested. They are ranked by $a / b$, and although no direct relationship to band gap is found, it can be seen on the left hand side that for $a / b$ greater than 0.3 , the approximate material band gap is less than or equal to 1.1 . On the right hand side, where $a / b$ is less than 0.2 , approximate band gap (where known) is greater than 1.4. This suggests that the latter values of $a / b$ and band gap may be appropriate for IPV; in general, lowest $a / b$ is recommendable for IPV.

Two distinct technological groups are also found when ranking the results with respect to $a$ : those cells with a value of $a$ greater than 1.4 (left-hand side of Table 2, including monocrystalline Silicon, polycrystalline Silicon and CIGS) and those where $a$ was in the range $0.17-0.74$ (on the right hand side, amorphous Silicon, CdTe, Gallium compounds and dye cells). These two modes have already been identified [6] and this is the first time that numerical variables have been associated with them. However, neither $a$ nor $a / b$ sort cells exactly by band gap. This can be explained not only due to the testing of both cells and modules, but also to the variety of processes and methods used to produce the samples.

Table 3 shows the parameters of equation 9 for the results in Figure 3 . These are then compared in Table 4 with the values under AM1.5 (Table 2). As can be seen in Table 4, the amorphous Silicon parameters increase by $13-34 \%$. The dye cell samples parameter $b$ changes little and have an $18-36 \%$ increase in parameter $a$. The crystalline Silicon samples parameters decrease from $51-62 \%$. This indicates that the latter samples are affected more by spectral mismatch (product of the incident spectrum and the spectral response of the cell integrated over the response range) than the amorphous and dye cells.

Table 3: Phenomenological model parameters for $1-100 \mathrm{~W} / \mathrm{m}^{2}$ under the fluorescent

\begin{tabular}{|c|c|c|c|c|c|c|c|c|c|}
\hline Cell code (see Table 1) & $a_{F}$ & $\overline{b_{F}}$ & $\mathbf{R}^{2}$ & $a_{F} / b_{F}$ & Cell code (see Table 1) & $a_{F}$ & $\overline{b_{F}}$ & $\mathbf{R}^{2}$ & $\overline{a_{F} / b_{F}}$ \\
\hline XSi-BP & 0.82 & 1.26 & 1.00 & 0.65 & aSi-Sino & 0.46 & 2.76 & 1.00 & $\overline{0 .}$ \\
\hline xSi-EdSi & 0.57 & 0.95 & 1.00 & 0.60 & aSi-Tess & 0.57 & 6.11 & 1.00 & 0 . \\
\hline \multicolumn{5}{|c|}{$\mathrm{Nb}: a_{\mathrm{F}} \& b_{\mathrm{F}}$ are values of $a \& b$ under fluo. source } & PC-IPC2 & 0.57 & 4.26 & 1.00 & 0 . \\
\hline \multicolumn{3}{|l|}{ Average $R^{2}$ for all 6 samples } & 1.00 & & PC-GCSA & 0.48 & 4.29 & 0.99 & 0 . \\
\hline
\end{tabular}


Table 4: The ratio of fluorescent parameters (Table 3) to AM1.5 parameters (Table 2)

\begin{tabular}{lcc} 
Cell code (see Table 1) & $\boldsymbol{a}_{\mathrm{F}} / \boldsymbol{a}_{\mathrm{AM}}$ & $\boldsymbol{b}_{\mathrm{F}} / \boldsymbol{b}_{\mathrm{AM}}$ \\
\hline xSi-BP & 0.48 & 0.49 \\
xSi-EdSi & 0.41 & 0.33
\end{tabular}

\begin{tabular}{lcc} 
Cell code (see Table 1) & $\boldsymbol{a}_{\mathrm{F}} / \boldsymbol{a}_{\mathrm{AM}}$ & $\boldsymbol{b}_{\mathrm{F}} / \boldsymbol{b}_{\mathrm{AM}}$ \\
\hline aSi-Sino & 1.15 & 1.34 \\
aSi-Tess & 1.32 & 1.13 \\
PC-IPC2 & 1.36 & 1.00 \\
PC-GCSA & 1.18 & 0.98
\end{tabular}

\section{Discussion}

The effect of series resistance $\left(R_{S}\right)$ and parallel (shunt) resistance $\left(R_{P}\right)$ on solar cell $I / V$ curves is known [13], see equivalent circuit in Figure 6 and modelled [14]. At high $\mathrm{E}_{\mathrm{rad}}$ intensity, high $R_{S}$ reduces $F F$ whilst at low $E_{r a d}$, low $R_{P}$ reduces $F F$. The effect of $R_{S}$ and $R_{P}$ on efficiency can be seen in the gradient of the curves in Figure 2. This is consistent with the hypothesis [15] that where $\mathrm{R}_{\mathrm{S}}$ is sufficiently high that the shunt current is negligible, then efficiency below 1 sun will first increase as radiant energy intensity is reduced. As can be seen, some samples in the intensity decade $100-1000 \mathrm{~W} / \mathrm{m}^{2}$ have such a negative gradient. Where the gradient approaches zero, the efficiency is maximised. For IPV products in particular, knowledge of these efficiency variations can improve product and cell design as well as help to explain variations in performance of ostensibly similar modules.

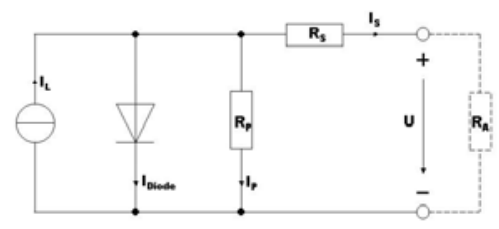

Figure 6: Equivalent circuit of Photovoltaic solar cell or module

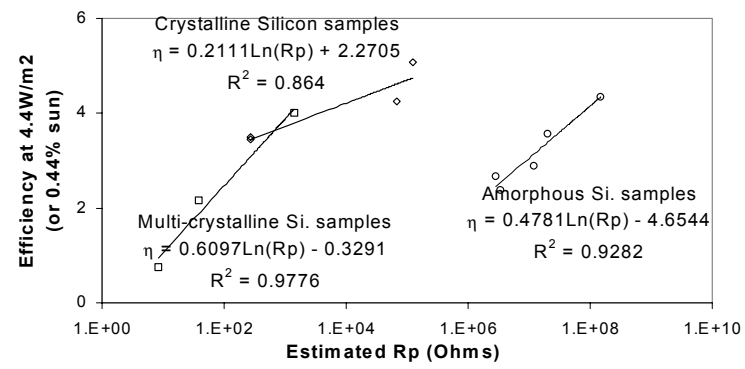

Figure 7: $\mathrm{R}_{\mathrm{SC}}$ (approx. $\mathrm{R}_{\mathrm{P}}$ ) vs. efficiency under low illumination $\left(4.4 \mathrm{~W} / \mathrm{m}^{2}\right.$ filtered AM1.5)

As a complement to the results, it would be interesting to test further cell samples, especially of those technologies which are not as well represented here (e.g. CIGS) and those that performed well at low light levels, such as CdTe and the Gallium compounds. This would allow further scrutiny and improvement of the model presented. An ideal outcome from the IPV practitioner perspective would be a model based on easily accessible data (such as 1 sun efficiency) which would provide a prediction of cell performance over the full range of intensities tested here. The phenomenological model (equation 8) is valid for only some of the samples across the full range tested, $0.8-1000 \mathrm{~W} / \mathrm{m}^{2}$, namely Solems, BP Millenium and Tessag. In order to extend the validity, more terms are required to model that part of the efficiency-intensity curve where the gradient becomes negative. It is also necessary to investigate the physical meaning of the control parameters $a$ (equation 10) and $b$ (equation 11). 
Another area requiring better understanding is the physical mechanism which induces $R_{P}$. Assuming it is not dependent on light intensity, the $\mathrm{R}_{\mathrm{P}}$ at low light intensities can be related to efficiency. $R_{P}$ was estimated by taking the $R_{S C}$ for a selection of samples at a low $E_{\text {rad }}$ $\left(4.4 \mathrm{~W} / \mathrm{m}^{2}\right)$. Figure 7 suggests that there is a natural $\log$ relationship between $R_{P}$ and efficiency per technology.

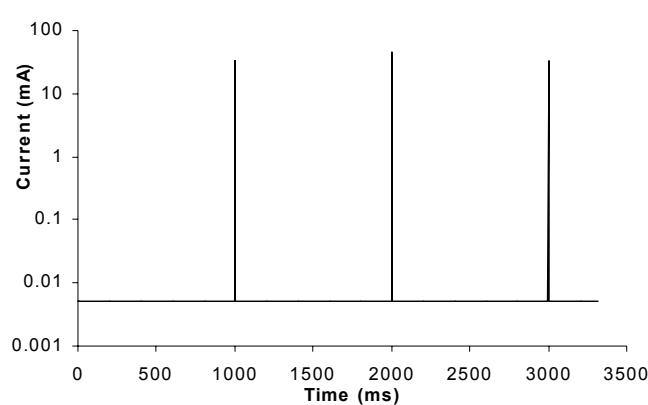

Figure 8: Typical charge consumption of datalogging or sensor device

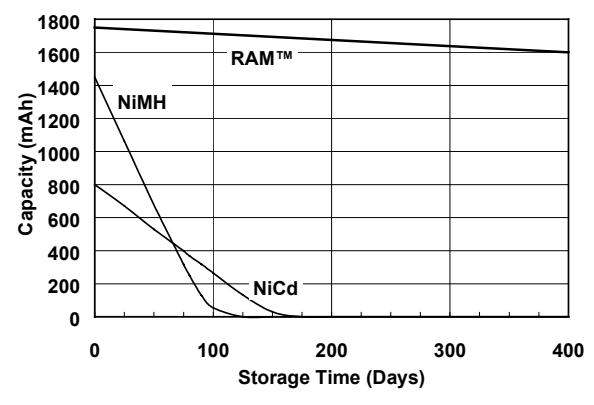

Figure 9: Secondary cell self-discharge technology comparison, courtesy Battery Technologies Inc., CAN

The effect of incident radiant energy spectrum is of interest for the IPV practitioner. It has been demonstrated in Figure 3 that amorphous Silicon sample efficiency was higher with fluorescent spectrum (up $14-34 \%$ at $2 \mathrm{~W} / \mathrm{m}^{2}$ ) whilst the crystalline Silicon samples efficiency deteriorated (down $51-57 \%$ at $2 \mathrm{~W} / \mathrm{m}^{2}$ ) compared with filtered AM1.5. It would be interesting to test other samples as well as corroborate the results with comparison of the spectral response. The latter was not pursued, as the selection of samples did not include a single cell sample (a pre-requisite for the spectral response equipment) for each technology. For those interested in developing IPV products, other factors apart from PV design are important. Ideal products that can benefit from PV power perform datalogging and/or sensor functions. This is because their functionality is only required in intermittent spikes separated by relatively long "rest" periods such as Figure 8.

The main charge consumption need is therefore the standby current, which unfortunately for many present IC designs can be of the order of $\mathrm{mA}$ rather than the $\mu \mathrm{A}$ typically produced indoors by PV. The development of ever more efficient micro-controller standby/sleep modes is important for PV penetration in indoor products.

Most IPV products require charge storage to allow use when $E_{\text {rad }}$ is too low. A number of charge storage technologies are available, one of which $\left(\mathrm{RAM}^{\mathrm{TM}}\right)$ is well adapted to IPV due to its low rate of self-discharge (see Figure 9).

Further aspects of IPV design that should be considered include final appearance, solar cell orientation with respect to the $E_{\text {rad }}$ source(s) as well as a number of module design parameters such as cell width [16]. 


\section{Conclusion}

At the beginning of this paper, the lack of comparable low intensity solar cell data was mentioned. This gap has been partly filled by the results presented here for 21 samples representing 8 technologies tested under two spectra types. These show that efficiency is not constant with intensity, technology and spectra.

Also the reasons why existing solar cell comparisons e.g. [1] are not applicable for indoor PV (IPV) design have been presented. For example, absolute efficiency may vary markedly with intensity in the decades $1-100 \mathrm{~W} / \mathrm{m}^{2}$ (see Figure 2).

The lack of appropriate models has also been identified; two forms of a phenomenologically based model have been shown to correlate well to experimental results in the intensity range of interest for IPV design.

This paper has identified a number of issues important to IPV design and has provided results that may contribute to better resolving them.

\section{Acknowledgements}

W. Durisch (PSI, CH), G. Leyland, M-O. Hongler, F. Dusonchet (all 3 at EPFL, CH) and E. Meyer (University of Port Elizabeth, South Africa) are thanked for helpful discussion. We are grateful to C. Droz (University of Neuchatel, CH), R. Gottschalg (CREST, UK), S. Kurtz (NREL, CO., USA) and D. Baetzner (LSSP, Zurich, CH) for corrections. Above all, our thanks to all those who provided samples.

\section{References}

[1] IEC-904-3, IEC Standard (1989) i.e. $1000 \mathrm{~W} / \mathrm{m}^{2}$ AM1.5 normal to cell surface at $25^{\circ} \mathrm{C}$

[2] Bücher K., Solar Energy Materials and Solar Cells, Volume 47,1997, pp. 85-94

[3] Roth W., Schmid J., 8th EC PhotovoltaicSolar Energy Conference pp. 263-9

[4] Pettersson H. et al., Electronics Goes Green 2000+, Berlin pp. 735-739

[5] Nakajima H., JEE June 79 p26-29

[6] Randall, J.F., et al. (2001) 17th EPVSECE, Munich

[7] Camani M. et al., 2nd World Conf. and Ex. on PVSEC 6-10 July 1998, Vienna

[8] Eikelboom J.A., Jansen M.J., ECN report ECN-C--00-067 June 2000

[9] Anderson D., Bishop J., Dunlop E.,16th EPVSECE 1-5 May 2000, Glasgow

[10] Chianese D., et al, 16th EPVSECE 1-5 May 2000, Glasgow

[11] Software available on 31.1.02 via http://radsite.lbl.gov/radiance/HOME.html

[12] Möller, H. J., Semiconductors for Solar Cells (1993) Artech pp. 29 Equation 2.39

[13] Green M., Solar Cells Operating Principles, (1982) NSW pp. 88

[14] Oritz-Conde A. et al, Solid-State Electronics 44 (2000) pp.1861-1864

[15] Meyer E.L, MSc Thesis, Uni. of Port Elizabeth, South Africa, Chap. 3 pp. 15-30

[16] Burgleman M., Niemegeers A., Sol. En. Mat. and Sol. Cells, Vol. 57,1999, pp. 85-95 